\title{
Aortic Valve Replacement with Bovine Pericardium in Patients with Aortic Valve Regurgitation A Single-Center Experience
}

\author{
Weiyong Sheng, ${ }^{1 *} \mathrm{MD}$, Guochang Zhao,${ }^{1 *} \mathrm{MD}$, Yangyang Chao, ${ }^{1} \mathrm{MD}$, Fuqiang Sun, ${ }^{1} \mathrm{MD}$, \\ Zhouyang Jiao, ${ }^{1}$ MD, Penghong Liu, ${ }^{1}$ MD, Hang Zhang, ${ }^{1} \mathrm{MD}$, Xingxing Yao, ${ }^{1}$ MD, \\ Fanfan Lu, ${ }^{1}$ MD, Haibo Zhan, ${ }^{1}$ MD, Jiawei Zhou, ${ }^{1}$ MD, Tao Song, ${ }^{1}$ MD, \\ Liang Tao, ${ }^{2} \mathrm{MD}$ and Chao Liu, ${ }^{1} \mathrm{MD}$
}

\begin{abstract}
Summary
This study aimed to analyze the early and mid-term outcomes of aortic valve replacement with bovine pericardium in the treatment of aortic valve regurgitation.

From January 2015 to March 2018, 36 patients (19 men; mean \pm standard deviation [SD] age, $46.70 \pm$ 16.60 years) underwent aortic valve replacement with bovine pericardium. The bovine pericardium was intraoperatively measured and shaped using an Ozaki template, according to the shape and size of the individual patient's aortic valve leaflets. Additional procedures were performed, including ventricular septal defect repair in 5 cases, mitral valve reconstruction in 6 cases, tricuspid valve reconstruction in 6 cases, and coronary artery bypass grafting in 3 cases.

There were no perioperative deaths. One elderly patient with postoperative respiratory failure recovered after symptomatic treatment. One patient with frequent ventricular tachycardia after intraoperative cardiac re-jump underwent intra-aortic balloon counterpulsation (IABP), and the IABP device was successfully removed on the second postoperative day. Within the first 6 months of follow-up, there were no death events, no reoperation events, and no additional thromboembolic events. Follow-up echocardiography was performed for 6 months, with average left ventricular ejection fraction of $62.01 \pm 3.21 \%$, mean transvalvular pressure gradient of $11.17 \pm$ $4.90 \mathrm{mmHg}$, and mean aortic valve velocity of $1.60 \pm 0.58 \mathrm{~m} / \mathrm{s}$. Compared with the preoperative transthoracic echocardiography findings, the results at the six-month follow-up were statistically significant $(P<0.05)$. Mild aortic valve regurgitation occurred in 2 patients $(5.56 \%)$, whereas other patients had no or only minimal aortic valve regurgitation $(n=34,94.44 \%)$. Moderate aortic valve regurgitation occurred in one patient at 9 months after the initial operation. This was found to be due to infective endocarditis, and a biological valve was finally implanted.

Aortic valve replacement with bovine pericardium in the treatment of aortic valve regurgitation is feasible, and good early and mid-term results are achieved. Long-term results need to be followed up in the future.
\end{abstract}

Key words: Ozaki, Autologous pericardium, Calcification

(Int Heart J 2019; 60: 1344-1349)

A ortic valve regurgitation is the only valvular heart disease that causes both increased left ventricular volume load and increased left ventricular endsystolic pressure load. ${ }^{1)}$ Aortic valve regurgitation is frequently caused by rheumatic disease (common in developing countries), congenital abnormalities (mainly bicuspid aortic valve), infective endocarditis, Marfan syndrome, ascending aortic dissection, and other conditions. ${ }^{1)}$ Nowadays, mitral and tricuspid valve repairs are becoming more popular than valve replacement because of their standardization and reproducibility. Nevertheless, valve re- placement is still the current classic surgical treatment and remains the gold standard recommended by guidelines for aortic valve diseases. ${ }^{2)}$ The recognition of the long-term problems of existing prostheses and the widespread acceptance of advanced repair techniques for atrioventricular valves have aroused the interest of many surgeons in the field of aortic valve repair. The crucial factor for determination of whether aortic valve repair is feasible is the quality of the aortic valve leaflets. When not available, the leaflet can be replaced with bovine or autologous pericardium or other biological materials. Replacement of all 3

From the ${ }^{1}$ Department of Cardiovascular Surgery, First Affiliated Hospital of Zhengzhou University, Zhengzhou, China and ${ }^{2}$ WuHan Asia Heart Hospital, WuHan, China.

*These authors contributed equally to this work.

Address for correspondence: Chao Liu, MD, Department of Cardiovascular Surgery, First Affiliated Hospital of Zhengzhou University, No. 1 Jianshe Road, Erqi District, Zhengzhou, Henan 450052, China. E-mail: liubeilun@me.com

Received for publication December 11, 2018. Revised and accepted April 9, 2019

Released in advance online on J-STAGE November 15, 2019.

doi: 10.1536/ihj.18-695

All rights reserved by the International Heart Journal Association. 

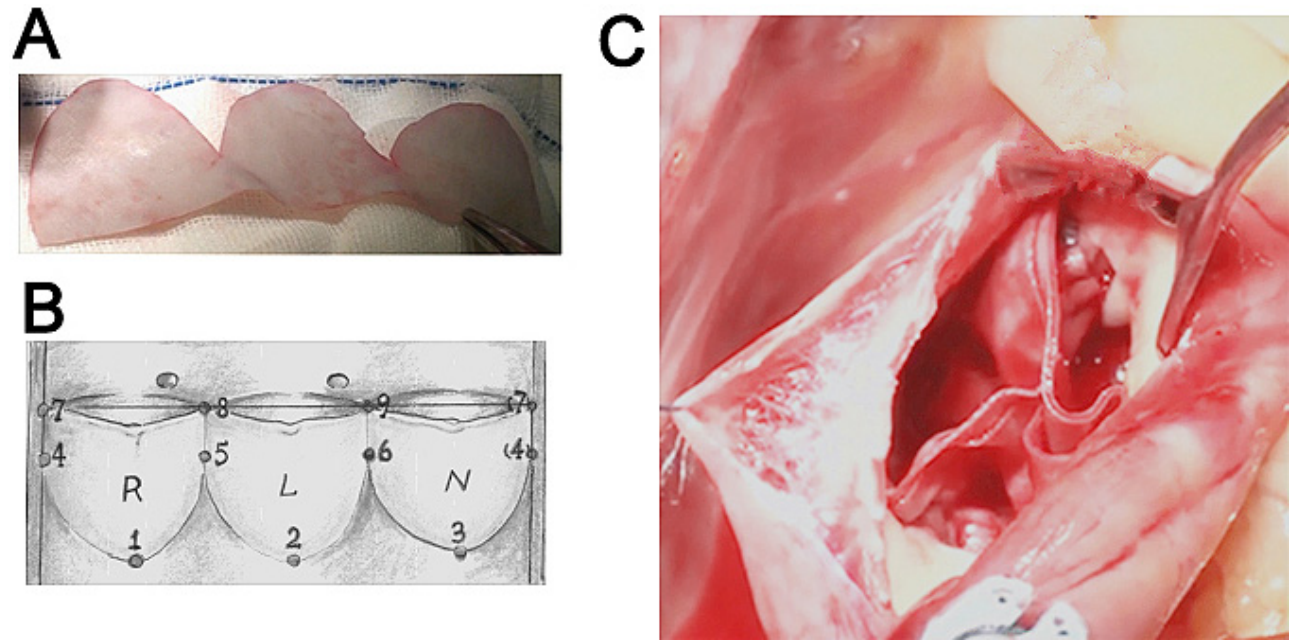

Figure. A: According to the template formula, the pericardium of the corresponding shape is drawn and cropped. B: Position the main suture position according to the 9-point method and suture from the center of the annulus. C: Completion of aortic valve reconstruction.

aortic cusps is technically more reliable than single cusp replacement because it is impossible to achieve 3 equal cusps when one or 2 of the cusps are made of different materials. ${ }^{3)}$ In 2011, Ozaki, et al. in their study described the technique of replacing the 3 aortic leaflets with glutaraldehyde-treated autologous pericardium and reported excellent long-term follow-up results in a large patient cohort. In their studies, the potential benefits of this technique included avoidance of anticoagulation, avoidance of foreign materials, and its suitability for patients with small aortic annuli or infective endocarditis. ${ }^{4,5)}$ This report reviews our initial experience with aortic valve replacement using bovine pericardium in patients with aortic valve regurgitation.

\section{Methods}

This clinical study was approved by the Institutional Review Board of the First Affiliated Hospital of the Zhengzhou University and was conducted in compliance with the Declaration of Helsinki. In January 2015, the first aortic valve replacement with bovine pericardium was performed in our clinical department. The indication for the operation was moderate to severe or severe aortic valve regurgitation. ${ }^{6}$ ) Data were collected from January 2015 to March 2018. The decision on whether or not to use this procedure was intensively discussed with each patient preoperatively, and all patients were informed of the surgical process before they signed informed consent. In our opinion, every patient who is suitable for a biological valve replacement or has a reason for avoiding oral anticoagulation is an appropriate candidate for this procedure, especially patients with economic difficulties. The contraindication was the coexistence of mitral valve disease that needs mechanical valve replacement. All operations were performed by the same surgical team. The primary endpoints were in-hospital mortality, reoperation, and thromboembolic events. Intraoperative and perioperative data, as well as six-month follow-up data (especially echocar- diographic data), were also collected and analyzed.

Surgical technique: All patients underwent transthoracic echocardiography (TTE) evaluation performed by the same sonographer before surgery. After general anesthesia induction through endotracheal intubation, all patients were routinely monitored using transesophageal echocardiography (TEE). TEE can improve the sensitivity and specificity for identifying abnormal leaflets compared with TTE. ${ }^{7}$ Except for the origin and pretreatment of the pericardium, the operative technique was the same with the Ozaki procedure, which has been previously reported. ${ }^{4)}$ In brief, all patients underwent standard cardiopulmonary bypass with ascending aortic and right atrium venous cannulation. Moderate hypothermia and a left ventricular vent were used. Antegrade cold blood cardioplegia was used for myocardial protection. Repeated doses of cardioplegia were given, if required, at 30-minute intervals throughout the operation. After a transverse aortotomy, the diseased cusps were resected. The distance between each commissure was measured with a measuring device. Correct measurement is vital, as it directly affects the size and shape of the pericardium. Then, the bovine pericardium (Beijing Balance Bio-Engineering Co., Ltd., Beijing, China) was subsequently cut according to the Ozaki template that corresponded to the measured size (Figure A). The annular margins of the prepared bovine pericardium leaflets were sutured to the aortic annulus by using 4-0 polypropylene (Prolene 4-0; Ethicon, Somerville, NJ, USA).

All sutures were knotted and reinforced with a pericardium patch outside the aorta. Before closing the incision, the functionality of the substituted valve was reassessed with water testing. Before removing the aortic cross-clamp, the pressure generated by re-infusion of warm blood antegrade cardioplegia provided an estimate of the valve function. After weaning from cardiopulmonary bypass, intraoperative TEE was used to estimate the early function of the repaired valve. In 25 patients, an isolated aortic valve replacement was performed. The re- 
maining 11 patients underwent additional procedures, including 5 cases of ventricular septal defect repair, 6 cases of mitral valve reconstruction, 6 cases of tricuspid valve reconstruction, and 3 cases of coronary artery bypass grafting.

The patients were routinely monitored in the intensive care unit (ICU) postoperatively. Patients with isolated aortic valve replacement were not administered with anticoagulation therapy. Anticoagulation was provided to patients with combined valve reconstruction, and antiplatelet therapy was prescribed to patients with combined coronary artery bypass grafting.

\section{Results}

Preoperative general data: Between January 2015 and March 2018, a total of 36 patients were operated on at our center. The general data of the patients are presented in Table I. All patients were adults. The mean \pm standard deviation (SD) age was $46.70 \pm 16.60$ years, and $61.29 \%$ $(n=19)$ of the patients were men. The mean EuroSCORE II score was $2.14 \pm 1.29$.

Four patients had moderate to severe aortic regurgita-

Table I. General Data $(n=36)$

\begin{tabular}{lc}
\hline \multicolumn{1}{c}{ Variable } & $46.70(16.60)$ \\
\hline Age in years, mean (SD) & $19(52.78)$ \\
Male sex, $n(\%)$ & $25.52(4.9)$ \\
Body mass index in $\mathrm{kg} / \mathrm{m}^{2}$, mean (SD) & $1.71(1.2)$ \\
Body surface area in $\mathrm{m}^{2}$, mean (SD) & $5(13.89)$ \\
Diabetes mellitus, $n(\%)$ & $11(30.56)$ \\
Dyslipidemia, $n(\%)$ & $15(41.67)$ \\
Arterial hypertension, $n(\%)$ & $8(22.22)$ \\
Active smoking, $n(\%)$ & $25(69.44)$ \\
Pure aortic valve regurgitation, $n(\%)$ & $19(52.77)$ \\
Rheumatic cause, $n(\%)$ & $11(30.55)$ \\
Congenital cause, $n(\%)$ & $6(16.66)$ \\
Active endocarditis cause, $n(\%)$ & $3(8.33)$ \\
Coronary heart disease, $n(\%)$ & $2(5.56)$ \\
Dyspnea NYHA I, $n(\%)$ & $4(11.11)$ \\
Dyspnea NYHA II, $n(\%)$ & $29(80.56)$ \\
Dyspnea NYHA III, $n(\%)$ & $1(2.77)$ \\
Dyspnea NYHA IV, $n(\%)$ & $3(8.33)$ \\
COPD, $n(\%)$ & $1(2.77)$ \\
Chronic renal disease, $n(\%)$ & $1(2.77)$ \\
Dialysis, $n(\%)$ & $2.14(1.29)$ \\
EuroSCORE II in \%, mean (SD)
\end{tabular}

COPD indicates chronic obstructive pulmonary disease; NYHA, New York Heart Association; and SD, standard deviation. tion, and the remaining patients had severe regurgitation. The classification according to the etiology of the disease was as follows: 19 cases of rheumatic disease; 11 cases of congenital abnormalities; and 6 cases of infective endocarditis. There were 25 patients with pure aortic valve regurgitation, and 11 patients had additional diseases, including 5 cases of ventricular septal defect, 6 cases of mitral valve regurgitation, 6 cases of tricuspid valve regurgitation, and 3 cases of concomitant coronary heart disease. Angina pectoris class II was observed in 2 patients $(5.55 \%)$ and angina pectoris class III in one patient $(2.77 \%)$. A total of 29 patients $(80.56 \%)$ had dyspnea of the New York Heart Association (NYHA) class III, and one patient $(2.77 \%)$ had dyspnea of NYHA class IV. NYHA class II and I dyspnea occurred in $4(11.11 \%)$ and $2(5.55 \%)$ patients, respectively.

Preoperative echocardiographic results: The preoperative echocardiographic results of the 36 patients are presented in Table II. The preoperative TTE showed a mean aortic valve velocity of $2.18 \pm 1.08 \mathrm{~m} / \mathrm{second}$ and a mean transvalvular pressure gradient of $27.12 \pm 14.06 \mathrm{mmHg}$. The average diameter of the annulus was $24.9 \pm 2.4 \mathrm{~mm}$. The mean \pm SD preoperative left ventricular ejection fraction (LVEF) was $58.03 \% \pm 11.03 \%$. There were 26 patients $(72.22 \%)$ with tricuspid aortic valve and 10 patients $(27.78 \%)$ with bicuspid aortic valve.

Intraoperative data: The intraoperative data are summarized in Table III. In all patients, the mean aortic crossclamp time was $101.17 \pm 25.76$ minutes and the mean cardiopulmonary bypass time was $121.35 \pm 38.52$ minutes. In patients with isolated aortic valve replacement procedures, the mean aortic cross-clamp time was $90.28 \pm$ 19.23 minutes and the mean cardiopulmonary bypass time was $114.26 \pm 39.46$ minutes. In patients with combined procedures, the mean aortic cross-clamp time was 116.12 \pm 25.21 minutes and the mean cardiopulmonary bypass time was $146.42 \pm 27.22$ minutes. The implant size of the coronary cusp varied from 23 to $35 \mathrm{~mm}$. The most frequent sizes were 25 and $29 \mathrm{~mm}$. Of the 36 patients, 25 $(69.44 \%)$ had an isolated aortic valve replacement procedure and $11(30.55 \%)$ had different additional procedures. These additional procedures included 5 cases of ventricular septal defect repair, 6 cases of mitral valve reconstruction, 6 cases of tricuspid valve reconstruction, and 3 cases of coronary artery bypass grafting.

Postoperative data: The postoperative data are summarized in Table IV. Postoperatively, the patients left the ICU after an average stay of $3.41 \pm 5.98$ days and were discharged from the hospital after a mean of $10.04 \pm 3.16$ days. Patients with isolated aortic valve replacement pro-

Table II. Preoperative Echocardiographic Results $(n=36)$

\begin{tabular}{lc}
\hline \multicolumn{2}{c}{ Variable } \\
\hline Left ventricular ejection fraction in \%, mean (SD) & $58.03(11.03)$ \\
Tricuspid aortic valve, $n(\%)$ & $26(72.22)$ \\
Bicuspid aortic valve, $n(\%)$ & $10(27.78)$ \\
Mean transvalvular aortic pressure gradient in mmHg, mean (SD) & $27.12(14.06)$ \\
Mean aortic valve velocity in m/second, mean (SD) & $2.18(1.08)$ \\
Average diameter of the annulus in mm, mean (SD) & $24.9(2.4)$ \\
\hline
\end{tabular}

SD indicates standard deviation. 
Table III. Intraoperative Data $(n=36)$

\begin{tabular}{lr}
\hline \multicolumn{2}{c}{ Variable } \\
\hline Mean aortic cross-clamp time (in minute) in isolated aortic valve replacement procedures, mean (SD) & $90.28(19.23)$ \\
Mean aortic cross-clamp time (in minute) in combined procedures, mean (SD) & $116.12(25.21)$ \\
Mean cardiopulmonary bypass time (in minute) in isolated aortic valve replacement procedures, mean (SD) & $114.26(39.46)$ \\
Mean cardiopulmonary bypass time (in minute) in combined procedures, mean (SD) & $146.42(27.22)$ \\
Isolated aortic valve replacement procedure, $n(\%)$ & $25(69.44)$ \\
Combined procedure, $n(\%)$ & $11(30.56)$ \\
\hline
\end{tabular}

SD indicates standard deviation.

Table IV. Postoperative Data $(n=36)$

\begin{tabular}{lr}
\hline \multicolumn{2}{c}{ Variable } \\
\hline Days in the intensive care unit, mean (SD) & $3.41(5.98)$ \\
Days in the intensive care unit in isolated aortic valve replacement procedures, mean (SD) & $2.39(2.04)$ \\
Days in the intensive care unit in combined procedures, mean (SD) & $4.75(7.96)$ \\
In-hospital days, mean (SD) & $10.04(3.16)$ \\
In-hospital days in isolated aortic valve replacement procedure, mean (SD) & $9.81(2.14)$ \\
In-hospital days in combined procedures, mean (SD) & $11.02(3.34)$ \\
LVEF (postoperative) in \%, mean (SD) & $59.89(7.31)$ \\
No or trace aortic regurgitation, $n(\%)$ & $34(94.44)$ \\
Mild aortic regurgitation, $n(\%)$ & $2(5.56)$ \\
Moderate or severe aortic regurgitation, $n(\%)$ & $0(0)$ \\
Mean transvalvular aortic pressure gradient in mmHg, mean (SD) & $13.75(5.89)$ \\
Mean aortic valve velocity in m/second, mean (SD) & $1.60(0.58)$ \\
Postoperative myocardial infarction, $n(\%)$ & $0(0)$ \\
Postoperative stroke, $n(\%)$ & $0(0)$ \\
Major bleeding requiring surgical revision, $n(\%)$ & $0(0)$ \\
Overall mortality after 30 days, $n(\%)$ & $0(0)$ \\
\hline
\end{tabular}

LVEF indicates left ventricular ejection fraction; and SD, standard deviation.

Table V. Clinical Endpoints after 6 Months (After the In-Hospital Period)

\begin{tabular}{lc}
\hline \multicolumn{2}{c}{ Variable } \\
\hline Completed the 6 months follow-up period, $n(\%)$ & $36(100)$ \\
Follow-up (days), mean (SD) & $208.15(38.9)$ \\
Death events, $n(\%)$ & $0(0)$ \\
Reoperation events, $n(\%)$ & $0(0)$ \\
Thromboembolic events, $n(\%)$ & $0(0)$ \\
\hline
\end{tabular}

SD indicates standard deviation.

cedure left the ICU after a mean of $2.39 \pm 2.04$ days and were discharged after $9.81 \pm 2.14$ days. On the other hand, patients with combined procedures left the ICU after a mean stay of $4.75 \pm 7.96$ days and were discharged after $11.02 \pm 3.34$ days. One elderly patient with postoperative respiratory failure recovered after symptomatic treatment. One patient with frequent ventricular tachycardia after intraoperative cardiac re-jump underwent intraaortic balloon counterpulsation (IABP), and the IABP device was successfully removed on the second postoperative day. The postoperative echocardiographic follow-up evaluation before discharge showed a mean aortic valve velocity of $1.60 \pm 0.58 \mathrm{~m} / \mathrm{seconds}$ and a mean transvalvular pressure gradient of $13.75 \pm 5.89 \mathrm{mmHg}$. The mean \pm SD postoperative LVEF was $59.89 \% \pm 7.31 \%$. Only 2 patients developed mild regurgitation, and the remaining patients had no or only minimal aortic regurgitation before discharge. Patients with isolated aortic valve replacement were not administered with anticoagulation therapy. Anticoagulation therapy was provided to patients with combined valve reconstruction, and antiplatelet therapy was prescribed to patients with combined coronary artery bypass grafting.

Clinical endpoints: Patients were followed up through outpatient examinations or telephone interviews. The follow-up evaluation included symptoms, signs, NYHA classification of cardiac function, and echocardiography. Echocardiographic assessment was performed at one week, one month, 3 months, and every 6 months after the initial surgery. All patients completed 6 months of followup (Table V). There were no death events, no reoperation events, and no thromboembolic events within the first 6 months of follow-up. Moderate aortic valve regurgitation occurred in one patient at 9 months after the initial operation. This was found to be due to infective endocarditis, and a biological aortic valve was finally implanted in the patient.

Echocardiographic characteristics after 6 months: Follow-up echocardiography was performed for 6 months, and the results are presented in Table VI. The average LVEF was $62.01 \% \pm 3.21 \%$, the mean transvalvular pressure gradient was $11.17 \pm 4.90 \mathrm{mmHg}$, with the mean aortic valve velocity being $1.60 \pm 0.58 \mathrm{~m} / \mathrm{second}$. Compared with the preoperative TTE findings, the results at the six-month follow-up were statistically significant $(P<$ 0.05). Two patients $(5.56 \%)$ developed mild aortic regurgitation, whereas others had no or only minimal aortic re- 
Table VI. Echocardiographic Characteristics after 6 Months of Follow-Up $(n=36)$

\begin{tabular}{lc}
\multicolumn{2}{c}{ Variable } \\
\hline Completed the 6 months follow-up period, $n(\%)$ & $36(100)$ \\
LVEF in \%, mean (SD) & $62.01(3.21)$ \\
Aortic valve stenosis, $n(\%)$ & $0(0)$ \\
No or minimal aortic regurgitation, $n(\%)$ & $34(94.44)$ \\
Mild aortic regurgitation, $n(\%)$ & $2(5.56)$ \\
Moderate aortic valve regurgitation, $n(\%)$ & $0(0)$ \\
Mean transvalvular aortic pressure gradient in mmHg, mean (SD) & $11.17(4.90)$ \\
Mean aortic valve velocity in m/second, mean (SD) & $1.60(0.58)$ \\
\hline
\end{tabular}

LVEF indicates left ventricular ejection fraction; and SD, standard deviation.

gurgitation $(n=34,94.44 \%)$.

\section{Discussion}

Despite the limitations of biological valves in terms of durability and the disadvantages of complications associated with anticoagulation in the use of mechanical prostheses, aortic valve replacement is still the treatment of choice for acquired aortic valve diseases. Nowadays, mitral and tricuspid valve repairs are becoming more popular than valve replacement because of their standardization and reproducibility, as surgeons begin to focus on aortic valve repair. Aortic valve repair has a history of nearly 60 years. ${ }^{8)}$ The main repair methods include valve extension, annuloplasty, valve repair, junction incision, and leaflet cutting. ${ }^{9)}$ However, these types of conservative repair are not always feasible, especially for patients with calcified aortic valves. Simple decalcification, folding leaflets, and thinning leaflets have not shown good results. ${ }^{4}$

Since the late 1960s, many kinds of biological materials have been used to replace the aortic valve cusp tissue. ${ }^{10,11)}$ The use of the pericardium in cardiovascular surgery and specifically as a material for aortic valve repair is not new. The results from a large number of reports in recent years have been highly variable. ${ }^{12)}$ Aortic valve repair with autologous pericardium leaflet extension or replacement was described in the study by Duran, et al. ${ }^{13-15)}$ They treated the autologous pericardium with glutaraldehyde solution. The treated pericardium is used to increase the height of native aortic valve leaflets or just to replace the original aortic valve leaflets themselves. Glutaraldehyde treatment can provide more resistance against retraction and degeneration and can maintain the intrinsic pliability of the pericardial tissue. Odim, et al. reported that aortic valve repair with autologous pericardium leaflet extension or replacement was a good alternative for a congenitally diseased aortic valve. ${ }^{16)}$ In 2011 , Ozaki, et al. in their study reported replacing the 3 aortic valve leaflets with glutaraldehyde-treated autologous pericardium in a large patient cohort and achieved excellent long-term follow-up results. With total resection of all 3 diseased leaflets and replacement with an autologous pericardium, this procedure can be applied to any type of aortic valve disease. In their studies, the potential benefits of this technique included avoidance of anticoagulation, avoidance of foreign materials, and suitability for patients with small aortic annuli or infective endocarditis. ${ }^{4,5}$

However, further studies have found that although the autologous pericardium treated with glutaraldehyde has the advantages of sterility, good biocompatibility, no tissue rejection, and convenient material acquisition, ensuring the survival and growth of tissue cells, the residual aldehyde agent can cause degeneration of the pericardium tissue and sterile necrosis. Moreover, the pericardium calcifies easily and loses its growth ability, especially in the high-pressure intraluminal area of the aortic valve. ${ }^{17,18)}$ As a result, many surgeons hesitate in performing aortic valve replacement with glutaraldehyde-fixed autologous pericardium for fear of its long-term durability. Bovine pericardium is another option. Halees, et al. found no evidence of differences in structural valve deterioration between autologous pericardium and bovine pericardium in their 16year follow-up report on aortic valve reconstruction. ${ }^{3)}$ Nezhad, et al. in their study found that a higher freedom from reoperation during $72 \pm 42.5$ months of follow-up had no statistical significance when bovine pericardium was used $(95 \% \pm 5 \%)$ in comparison with autologous pericardium $(73 \% \pm 11 \%) .{ }^{19)}$ Evora, et al. reported the case of an operation on a patient with bovine pericardium cusp replacement, with good ventricular function and minimal structural valve deterioration at a long-term follow-up of 23 years. ${ }^{20)}$

Regardless of using autologous pericardium or bovine pericardium, pericardium calcification is a main cause for concern and determines the freedom from reoperation over the long-term. At our center, the bovine pericardium we used was the same as bovine pericardial bioprostheses, which have been found to be free of calcification for approximately 10 years. The traditional biological valve manufacturing process only consists of the crosslinking of biological valve leaflets with glutaraldehyde $(0.5 \%-0.625 \%)$. In this manner, the remaining unrinsed free glutaraldehyde easily combines with calcium to cause calcification, ultimately leading to tissue fatigue and loss of the cytoplasmic matrix. ${ }^{21)}$ Accordingly, the bovine pericardium we used was treated with hydroxychromium, and the hydroxychromium was combined with the remaining uncrosslinked glutaraldehyde to completely block the calcium ion binding site. An animal study has shown that this treatment can improve the long-term durability of the valve. ${ }^{22)}$ Song, et al. and Tao, et al. ${ }^{23,24}$ reported in their studies that this kind of bovine pericardium can be safely used for valve replacement in patients with aortic regurgitation, and they achieved encouraging mid-term outcomes. It is commercially available and can be purchased at a low price. With the use of bovine pericardium, the limita- 
tion of the quality of the patients' autologous pericardium can be overcome. Moreover, it eliminates the process of dissecting, fixing, and rinsing the autologous pericardium with glutaraldehyde.

This cohort of patients was followed up for 6-47 months, and none of the patients to date have had any fibrosis, contraction, or calcification of bovine pericardium. The lack of embolic events in the absence of anticoagulation and the applicability to any type of aortic valve disease were probably the main advantages of this technique. Together with its low cost, these features make bovine pericardium perfectly suited for use in developing countries. Continuous and careful follow-up of these patients is warranted.

Study limitations: The limitations of this study were the small sample size and the short follow-up duration. In addition, this study is not a case-control study. Prospective case-control studies are ideal for determining the effectiveness of new aortic valve reconstruction methods compared with other conventional aortic valve replacement or repair procedures. Long-term results should be evaluated and followed up for at least 15-20 years. At the same time, the long-term results of bovine pericardium and autologous pericardium or other novel biomaterials need to be given further attention.

\section{Acknowledgment}

The authors thank Beijing Balance Bio-Engineering Co., Ltd., for providing the bovine pericardium.

\section{Disclosure}

Conflicts of interest: None.

\section{References}

1. Brtko M. Aortic Regurgitation: From Diagnosis to Indication. In: Vojacek J, Zacek P, Dominik J, eds. Aortic Regurgitation. 1st ed. Hradec Kralove, Czech Republic: Springer International Publishing AG; 2018: 69-75.

2. Nishimura RA, Carabello B. Operationalizing the 2014 ACC/ AHA Guidelines for Valvular Heart Disease: A Guide for Clinicians. J Am Coll Cardiol 2016; 67: 2289-94.

3. Halees ZA, Shahid MA, Sanei AA, Sallehuddin A, Duran C. Up to 16 years follow-up of aortic valve reconstruction with pericardium: a stentless readily available cheap valve? Eur J Cardiothorac Surg 2005; 28: 200-5

4. Ozaki S, Kawase I, Yamashita $\mathrm{H}$, et al. Aortic valve reconstruction using self-developed aortic valve plasty system in aortic valve disease. Interact Cardiovasc Thorac Surg 2011; 12: 550-3.

5. Ozaki S, Kawase I, Yamashita H, et al. Aortic valve reconstruction using autologous pericardium for ages over 80 years. Asian
Cardiovasc Thorac Ann 2014; 22: 903-8.

6. Zamorano JL. The ESC Committee for Practice Guidelines (CPG). Euro Heart J 2013; 34: 703.

7. Chen J, Lu S, Hu K, et al. Clinical Characteristics and Surgical Treatment of Infective Endocarditis With Bicuspid Aortic Valve. Int Heart J 2017; 58: 220.

8. Lillehei CW, Gott VL, Dewall RA, Varco RL. Surgical treatment of stenotic and regurgitant lesions of the mitral and aortic valves by direct vision utilizing a pump oxygenator. J Thorac Surg 1958; 35: 154-91.

9. Duran CM, Alonso J, Gaite L, et al. Long-term results of conservative repair of rheumatic aortic valve insufficiency. Eur J Cardiothorac Surg 1988; 2: 217-23.

10. Bjoerk VO, Hultquist G. Teflon And Pericardial Aortic Valve Prostheses. J Thorac Cardiovasc Surg 1964; 47: 693.

11. Senning A. Fascia lata replacement of aortic valves. J Thorac Cardiovasc Surg 1967; 54: 465-70.

12. Duran CM, Gometza B, Shahid M, Al-Halees Z. Treated bovine and autologous pericardium for aortic valve reconstruction. Ann Thorac Surg 1998; 66: S166-9.

13. Beyersdorf F. Long-term results of conservative repair of rheumatic aortic valve insufficiency. Eur J Cardiothorac 1988; 2: 217-23.

14. Duran CM, Gometza B, Kumar N, Gallo R, Bjornstad K. From aortic cusp extension to valve replacement with stentless pericardium. Ann Thorac Surg 1995; 60: 428-32.

15. Duran CM, Gometza B, Kumar N, Gallo R, Martin-Duran R. Aortic valve replacement with freehand autologous pericardium. J Thorac Cardiovasc Surg 1995; 110: 511-6.

16. Odim J, Laks H, Allada V, Child J, Wilson H, Gjertson D. Results of aortic valve-sparing and restoration with autologous pericardial leaflet extensions in congenital heart disease. Ann Thorac Surg 2005; 80: 647-53.

17. Yu S, Okada Y, Nasu M, et al. Late results of mitral valve repair with glutaraldehyde-treated autologous pericardium. Ann Thorac Surg 2013; 95: 2000-6.

18. Evans CF, Defilippi CR, Shang E, Griffith BP, Gammie JS. Fresh autologous pericardium for leaflet perforation repair in mitral valve infective endocarditis. J Heart Valve Dis 2013; 22: 560-6.

19. Nezhad ZM, Kerchove LD, Hechadi J, et al. Aortic valve repair with patch in non-rheumatic disease: indication, techniques and durability. Eur J Cardiothorac Surg 2014; 46: 997-1005.

20. Evora PR, Arcêncio L, Evora PM, Menardi AC, Chaud F. Bovine pericardial patch augmentation of one insufficient aortic valve cusp with twenty-three-year positive clinical follow-up independent of the patch degeneration. Braz J Cardiovasc Surg 2017; 32: 49-52.

21. Biedrzycki LM, Lerner E, Levy RJ, Schoen FJ. Differential calcification of cusps and aortic wall of failed stented porcine bioprosthetic valves. J Biomed Mater Res 1997; 34: 411-5.

22. Cunanan CM, Cabiling CM, Tan TD, et al. Tissue characterization and calcification potential of commercial bioprosthetic heart valves. Ann Thorac Surg 2001; 71: S417-21.

23. Song L, Hua Z, Chen X, et al. Single cusp replacement in patients with ventricular septal defect and aortic insufficiency. J Card Surg 2015; 30: 520-4.

24. Tao L, Zeng XJ, Lim YP. Single cusp replacement for aortic regurgitation. Ann Thorac Surg 2008; 85: 946-8. 